\title{
Finite Element Modeling of Stress Evolution in Sn Films due to Growth of the $\mathrm{Cu}_{6} \mathrm{Sn}_{5}$ Intermetallic Compound
}

\author{
ERIC BUCHOVECKY ${ }^{1,2}$ NITIN JADHAV,${ }^{1}$ ALLAN F. BOWER, ${ }^{1}$ \\ and ERIC CHASON ${ }^{1}$ \\ 1.-Division of Engineering, Brown University, Providence, RI 02666, USA. 2.-e-mail: \\ buchovecky@brown.edu
}

\begin{abstract}
We use finite element simulations to quantitatively evaluate different mechanisms for the generation of stress in Sn films due to growth of the $\mathrm{Cu}_{6} \mathrm{Sn}_{5}$ intermetallic phase at the $\mathrm{Cu}-\mathrm{Sn}$ interface. We find that elastic and plastic behavior alone are not sufficient to reproduce the experimentally measured stress evolution. However, when grain boundary diffusion is included, the model results agree well with experimental observations. Examination of conditions necessary to produce the observed stresses provides insight into potential strategies for minimizing stress generation and thus mitigating Sn whisker growth.
\end{abstract}

Key words: $\mathrm{Pb}$-free solder, $\mathrm{Sn}$ whisker, finite element, grain boundary diffusion

\section{INTRODUCTION}

The growth of long, single-crystal whiskers from the surface of thin Sn coatings on $\mathrm{Cu}$ conductors was first observed over 50 years ago. ${ }^{1}$ Since Sn coatings are highly desirable on copper electronic interconnections as a means to reduce oxidation of the conductors and improve solderability, the potential for circuit failure due to whisker growth poses a significant threat to reliability. The problem was largely eliminated when it was discovered that alloying Sn with a small fraction of $\mathrm{Pb}$ prevented whisker formation. ${ }^{2,3}$ However, with the recent adoption of $\mathrm{Pb}$-free manufacturing practices in the electronics industry, whisker growth from Sn-plated $\mathrm{Cu}$ conductors has re-emerged as a significant threat, particularly in high-reliability applications.

The importance of compressive stress in Sn films as a primary driving force for whisker growth is well established. ${ }^{3,4}$ This stress can result from mechanical deformation during processing and handling of components, thermal mismatch, or in the case of $\mathrm{Sn}$ films on $\mathrm{Cu}$, the spontaneous growth of intermetallic compounds (IMCs). The latter process is of particular concern as it is difficult to control and can

(Received March 16, 2009; accepted July 23, 2009;

published online August 12, 2009) continue over long periods of time. A fundamental understanding of the process of stress generation in Sn films could potentially provide insight into strategies to minimize stress and thereby mitigate whisker growth. However, there is no clear consensus regarding the mechanisms by which localized growth of IMCs produces stress within the Sn film. ${ }^{5-14}$

In order to understand how stress develops in response to IMC growth, it is necessary to identify the mechanical deformation processes operating in the surrounding Sn film. Recent experimental work provides evidence of extensive dislocation activity within the Sn film, especially in the neighborhood of IMC grains, ${ }^{13,14}$ indicating that the Sn grains yield plastically. This conclusion is also consistent with reported stress measurements within the Sn layer which are very near the nominal yield stress for $\mathrm{Sn}$ $\left(14.5 \mathrm{MPa}^{15}\right)$ and remain relatively constant over time. ${ }^{7,11,13,16}$ In addition, self-diffusion along grain boundaries within $\mathrm{Sn}$ is assumed to provide a fast pathway for long-range transport. ${ }^{5,17}$ The role played by these plastic deformation processes in the development of stress within Sn films, however, has not been quantitatively assessed.

In this paper, we present a series of finite element simulations that allow us to quantitatively evaluate different mechanisms for the generation of stress in 
Sn films due to growth of the $\mathrm{Cu}_{6} \mathrm{Sn}_{5}$ intermetallic compound at the $\mathrm{Cu}$-Sn interface. Specifically, we examine the role of elastic and plastic deformation within Sn grains and the role of stress-driven diffusion of Sn along grain boundaries. It should be noted that we do not address the nucleation of whiskers, nor include the effect of local stress relaxation in the region surrounding a whisker. Also, for the purpose of this study we limit attention to stress generation in as-deposited Sn films and do not consider the effect of solder reflow, which can significantly alter the microstructure, thickness, and morphology of the Sn.

Our simulations show that elastic deformation and dislocation-mediated plastic flow alone are not sufficient to reproduce the experimentally measured relationship between stress and IMC volume. However, when grain boundary diffusion is included, the model results agree well with experimental observations. Examination of conditions necessary to produce the observed stresses provide insights into potential strategies for minimizing stress generation and thus mitigating whisker growth.

\section{EXPERIMENTAL BACKGROUND}

The relationship between IMC growth and film stress has been investigated through a series of experiments which allow simultaneous measurement of IMC volume and stress in the Sn for the same samples (presented previously by Chason et al. ${ }^{13}$ ). Here, we briefly review those results, calling attention to several key features. Figure 1 shows measured IMC volume per area and the corresponding average stress in the Sn layer obtained for a $1.45 \mu \mathrm{m}$ Sn film electroplated over $0.6 \mu \mathrm{m}$ of $\mathrm{Cu}$. It is important to note that the measured stress represents mean biaxial stress, i.e., the average of the two in-plane normal stress components, $\left(\sigma_{11}+\sigma_{22}\right) / 2$, integrated over the thickness of the Sn layer.

Examining the plot of IMC growth over time (Fig. 1a), IMC volume per area is seen to increase monotonically. The corresponding stress in the Sn, shown in Fig. 1b, is initially tensile but quickly becomes compressive. The stress reaches a maximum compressive value of about -12 MPa after approximately $20 \mathrm{~h}$, then remains relatively constant even though IMCs continue to grow. The observation that the average stress appears to attain a "steady-state" value indicates that further IMC growth is accommodated by processes that produce little additional stress within the Sn, such as plastic yield.

To understand how stress is generated by IMC growth, it is necessary to consider the nature of the IMC transformation reaction. At room temperature, $\mathrm{Cu}$ and $\mathrm{Sn}$ react irreversibly to form the $\mathrm{Cu}_{6} \mathrm{Sn}_{5}$ IMC. Scanning electron microscopy (SEM) and transmission electron microscopy (TEM) analyses reveal that IMC particles nucleate within the Sn at the $\mathrm{Cu}-\mathrm{Sn}$ interface, typically with greater probability at the triple junctions where grain boundaries

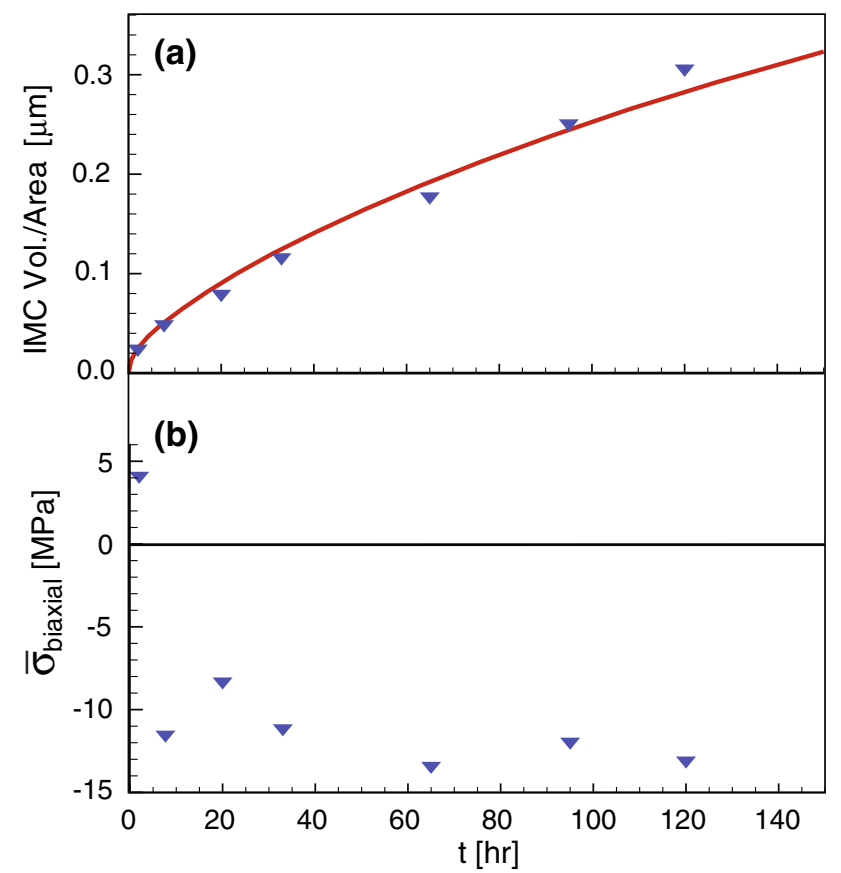

Fig. 1. Experimental measurements (symbols) of (a) IMC volume per area, and (b) average stress within the Sn layer for samples with $1.45 \mu \mathrm{m}$ pure $\mathrm{Sn}$ plated on $0.6 \mu \mathrm{m} \mathrm{Cu}$. Measurements corresponding to the same time were obtained from the same sample. The empirically fit curve (solid line) in (a) was used to model IMC growth in the numerical simulations and has the form $V_{\text {IMC }} / A=$ $\left(0.014 \mu \mathrm{m} \mathrm{h}^{-0.6}\right) t^{0.6}$. Data previously published in Chason et al. ${ }^{13}$

between $\mathrm{Sn}$ grains meet the $\mathrm{Cu}$ interface. ${ }^{13,14,18}$ After nucleation, growth of the particles proceeds at the IMC/Sn interface, ${ }^{5,19}$ with IMC particles primarily growing up into the Sn film. ${ }^{13,14,16,18}$ TEM analysis also provides evidence of dislocation activity within the Sn layer, particularly near IMC particles. ${ }^{13,14}$ This suggests that IMC growth induces sufficient stress within the surrounding Sn grains to cause dislocation-mediated plastic deformation.

In the case of pure $\mathrm{Sn}$ on $\mathrm{Cu}$, SEM and TEM examination of cross-sections through the thickness of the film show that IMC growth occurs only at the Sn-Cu interface. ${ }^{13,14}$ There is no evidence of isolated IMC particles away from the $\mathrm{Sn}-\mathrm{Cu}$ interface, either within Sn-Sn grain boundaries or in the Sn grains themselves. Thus, a model for stress generation must explain how IMC growth that is restricted to the base of the Sn layer results in the development of stress throughout the thickness of the film.

Stress generation in the Sn layer is intimately related to the volume changes associated with the formation of $\mathrm{Cu}-\mathrm{Sn}$ intermetallic compounds. Here we consider only reaction of $\mathrm{Cu}$ and $\mathrm{Sn}$ to form $\mathrm{Cu}_{6} \mathrm{Sn}_{5}$, since this is the sole IMC phase observed at room temperature. ${ }^{5}$ As discussed above, $\mathrm{Cu}_{6} \mathrm{Sn}_{5}$ is observed to grow into the $\mathrm{Sn}$. This requires the transport of $\mathrm{Cu}$ to the IMC growth front, where it then reacts with the adjacent Sn. ${ }^{5,19}$ The net volume change associated with the reaction is therefore 
Table I. Density and Molar Volume Data. Note that the Molar Volume of $\mathrm{Cu}_{6} \mathrm{Sn}_{5}$ Corresponds to 1 mole of Atoms and is Based on the Composition of 5/11 (=0.45) Atomic Fraction Sn

\begin{tabular}{llll}
\hline Species & & Density & Molar Volume \\
\cline { 4 - 4 } $\mathrm{Cu}$ & & $8.96 \mathrm{~g} / \mathrm{cm}^{3}$ & $7.09 \mathrm{~cm}^{3} / \mathrm{mol}$ \\
$\mathrm{Sn}$ & $7.30 \mathrm{~g} / \mathrm{cm}^{3}$ & $16.3 \mathrm{~cm}^{3} / \mathrm{mol}$ \\
$\mathrm{Cu}_{6} \mathrm{Sn}_{5}$ & & $8.28 \mathrm{~g} / \mathrm{cm}^{3}$ & $10.7 \mathrm{~cm}^{3} / \mathrm{mol}$ \\
\hline
\end{tabular}

partitioned into two spatially separated volume changes: volume loss within the $\mathrm{Cu}$ layer and volume increase within the Sn layer. Calculation of the resulting strain within the Sn layer depends on the details of the transformation from Sn to IMC. While these details are not well understood, the consequences of two limiting cases can be explored.

Consider a fixed volume of $\mathrm{Sn}$ adjacent to a growing IMC particle. If all of the Sn atoms in this region rearrange to form $\mathrm{Cu}_{6} \mathrm{Sn}_{5}$ as $\mathrm{Cu}$ atoms arrive, then the region will undergo a large volumetric expansion. Referring to the molar volume data summarized in Table I, the initial Sn volume of $(0.45) V_{\mathrm{m}}^{\mathrm{Sn}}=7.4 \mathrm{~cm}^{3} / \mathrm{mol}$ is replaced by $V_{\mathrm{m}}^{\mathrm{IMC}}=$ $10.7 \mathrm{~cm}^{3} / \mathrm{mol}$, corresponding to a $45 \%$ volume increase. This value is an upper bound, since some of the IMC volume may also be accommodated by motion of the $\mathrm{Cu}-\mathrm{Sn}$ interface.

At the other extreme, it is possible for the IMC to occupy the same volume as the $\mathrm{Sn}$ it replaces if sufficient $\mathrm{Sn}$ is expelled as point defects during the transformation. The expelled Sn could either be accommodated by the surrounding Sn lattice or adjacent grain boundaries. Stress generated by the diffusion of expelled $\mathrm{Sn}$ along grain boundaries will be discussed in the "Results and Discussion" section. For the case of Sn point defects inserted into the surrounding Sn lattice, the extremely low bulk self-diffusivity for $\mathrm{Sn}$ of $D \approx O\left(10^{-18} \mathrm{~cm}^{2} / \mathrm{s}\right)^{15}$ would effectively limit the volumetric expansion to a region immediately surrounding the IMC particles. The resulting localized expansion would be very similar to that produced by expansion of the IMC itself. For the purpose of modeling stress evolution in the Sn film, we assume that the transformation of $\mathrm{Sn}$ to $\mathrm{Cu}_{6} \mathrm{Sn}_{5}$ produces a localized volumetric expansion of $45 \%$. Details of the numerical implementation are presented in the following section. The effect of volume loss within the $\mathrm{Cu}$ substrate is not considered here. Since the $\mathrm{Cu}$ film is constrained against lateral deformation by the underlying $\mathrm{Si}$ wafer, stress in the $\mathrm{Cu}$ layer has little influence on the stress in the Sn.

\section{SIMULATION DETAILS}

Our finite element simulations model a polycrystalline Sn film with columnar grain structure bonded to a $\mathrm{Cu}$ substrate, as shown in Fig. 2. The

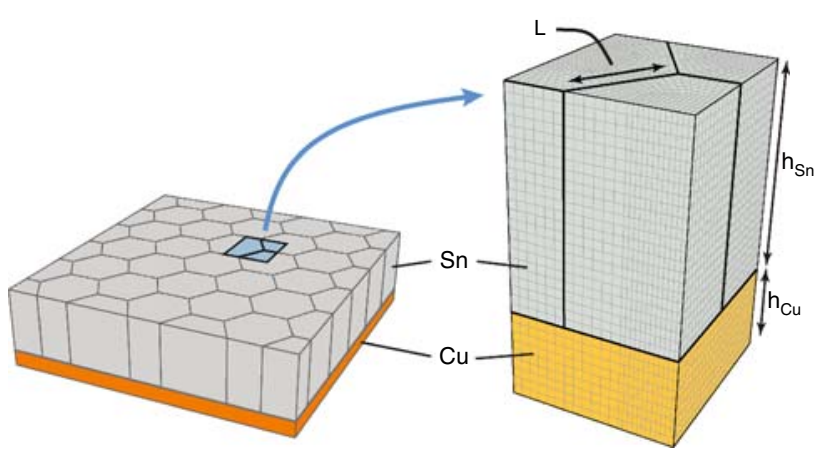

Fig. 2. The computational model used in the finite element simulations. The diagram on the left shows the $\mathrm{Cu}$-Sn bilayer film represented by the model. The $\mathrm{Sn}$ layer is comprised of columnar hexagonal grains separated by vertically oriented grain boundaries which can act as fast diffusion pathways. The highlighted region shows the outline of the actual model cell, which is shown in greater detail on the right. By applying symmetry boundary conditions on the lateral boundaries, the model cell simulates a perfectly regular film of infinite extent.

copper substrate has a thickness of $0.6 \mu \mathrm{m}$ and is treated as a homogeneous solid layer. The Sn film has a thickness of $1.45 \mu \mathrm{m}$ and consists of a periodic array of identical hexagonal grains measuring $0.75 \mu \mathrm{m}$ on a side (grain size is approximately $1.4 \mu \mathrm{m})$. The Sn grains are separated by vertically oriented grain boundaries which support stressdriven mass transport. We assume that flux of material out of the grain boundaries onto the free surface is prevented by the presence of a passivating oxide layer. Mechanical behavior of the oxide layer, however, is not included in the model. Symmetry boundary conditions are applied at the lateral boundaries of the model cell to effectively model an infinite film, and the base of the model cell is held fixed. All dimensions and parameters used in the simulations are listed in Table II.

The $\mathrm{Sn}$ grains and the $\mathrm{Cu}$ layer are treated as isotropic, elastic-plastic solids with Young's modulus $E_{\alpha}$, Poisson's ratio $v_{\alpha}$, and yield stress $Y_{\alpha}$ (where $\alpha$ is either $\mathrm{Sn}$ or $\mathrm{Cu}$ ). Plastic deformation within the solid elements is determined by application of the isotropic von Mises yield criterion $\left(J_{2}\right.$ plastic constitutive law). We assume a yield stress value under uniaxial tension for $\mathrm{Sn}$ of $14.5 \mathrm{MPa} .{ }^{15}$ While we recognize that the constitutive behavior of $\mathrm{Sn}$ is significantly more complex (exhibiting strong anisotropy in elastic and plastic deformation and stress relaxation via creep) the simplified behavior modeled here is sufficient to establish the mechanisms by which stress develops and is transmitted through the Sn film.

In addition to elastic and plastic deformation within the solids, we explicitly model stress-driven mass diffusion along the Sn-Sn grain boundaries, following the finite element formulation described by Bower and Wininger. ${ }^{24}$ The chemical potential driving the flux of atoms along a grain boundary is approximated as $\mu=-\Omega \sigma_{\mathrm{n}}$, where $\Omega$ is the atomic 
Table II. Parameters Used in the Simulations

\begin{tabular}{|c|c|c|c|}
\hline Quantity & Symbol & Value & Ref. \\
\hline Sn film thickness & $h_{\mathrm{Sn}}$ & $1.45 \mu \mathrm{m}$ & \\
\hline $\mathrm{Cu}$ film thickness & $h_{\mathrm{Cu}}$ & $0.6 \mu \mathrm{m}$ & \\
\hline Length of hexagonal grain edge & $L$ & $0.75 \mu \mathrm{m}$ & \\
\hline Elastic modulus of Sn & $E_{\mathrm{Sn}}$ & $50 \mathrm{GPa}$ & {$[15]$} \\
\hline Poisson's ratio of Sn & $v_{\mathrm{Sn}}$ & 0.36 & {$[15]$} \\
\hline Yield strength of $\mathrm{Sn}$ & $Y_{\mathrm{Sn}}$ & $14.5 \mathrm{MPa}$ & {$[15]$} \\
\hline Elastic modulus of $\mathrm{Cu}$ & $E_{\mathrm{Cu}}$ & $117 \mathrm{GPa}$ & {$[15]$} \\
\hline Poisson's ratio of $\mathrm{Cu}$ & $v_{\mathrm{Cu}}$ & 0.34 & {$[15]$} \\
\hline Yield strength of $\mathrm{Cu}$ & $Y_{\mathrm{Cu}}$ & $200 \mathrm{MPa}$ & {$[20]$} \\
\hline Elastic modulus of $\mathrm{Cu}_{6} \mathrm{Sn}_{5}$ & $E_{\mathrm{IMC}}$ & $86 \mathrm{GPa}$ & {$[21]$} \\
\hline Poisson's ratio of $\mathrm{Cu}_{6} \mathrm{Sn}_{5}$ & $v_{\mathrm{IMC}}$ & 0.30 & {$[21]$} \\
\hline Yield strength of $\mathrm{Cu}_{6} \mathrm{Sn}_{5}$ & $Y_{\text {IMC }}$ & $2000 \mathrm{MPa}$ & {$[22]$} \\
\hline Grain boundary diffusivity of Sn & $D_{\mathrm{gb}}$ & $4.8 \times 10^{-9} \mathrm{~cm}^{2} / \mathrm{s}$ & {$[23]$} \\
\hline Grain boundary width of Sn & $\delta$ & $0.5 \mathrm{~nm}$ & [23] \\
\hline Atomic volume of $\mathrm{Sn}$ & $\Omega$ & $0.027 \mathrm{~nm}^{3}$ & \\
\hline Temperature & $T$ & $298 \mathrm{~K}$ & \\
\hline
\end{tabular}

Note: $\mathrm{Cu}_{6} \mathrm{Sn}_{5}$ is not expected to deform plastically, but a yield strength is required in the model to allow transformation of Sn to IMC. $Y_{\text {IMC }}$ is given a very large value, consistent with experimental measurements. ${ }^{20}$

volume of $\mathrm{Sn}$ and $\sigma_{\mathrm{n}}$ is the normal stress acting across the plane of the grain boundary. The total flux of volume along the grain boundary [volume/ $(l \cdot t)]$ is then given by

$$
\overrightarrow{j_{\mathrm{v}}}=-\frac{\delta D_{\mathrm{gb}}}{k T} \nabla \mu \approx \frac{\Omega \delta D_{\mathrm{gb}}}{k T} \nabla \sigma_{\mathrm{n}},
$$

where $\delta$ is the grain boundary width, $D_{\mathrm{gb}}$ is the grain boundary diffusivity at temperature $T, k$ is Boltzmann's constant, and the gradient is taken in the plane of the grain boundary.

Differences in the normal stress across the grain boundary from one location to another lead to variations in the volume flux along the grain boundary. This nonuniform volume flux results in mass transport along the grain boundary: volume is removed from regions of more compressive normal stress and added to regions of less compressive normal stress. In the finite element formulation, the relative velocity of opposite faces of the grain boundary (normal to the grain boundary plane) is calculated from the divergence of the volume flux:

$$
\left[v_{\mathrm{n}}\right]=\nabla \overrightarrow{j_{\mathrm{t}}} \approx \frac{\Omega \delta D_{\mathrm{gb}}}{k T} \nabla^{2} \sigma_{\mathrm{n}}
$$

Thus, material particles on opposite sides of the grain boundary are displaced relative to one another, allowing local strain associated with stress-driven mass transport along grain boundaries to be explicitly modeled.

Stress is generated in our model by the growth of hemispherical IMC nodules up into the Sn from the $\mathrm{Cu}-\mathrm{Sn}$ interface (Fig. 3). These nodules grow radially into the Sn layer by the progressive transformation of adjacent Sn into IMC. In the simulations, the radius of the IMC nodules, $r_{\mathrm{IMC}}$, is increased incrementally over a series of time steps, at a rate specified to correspond to the experimentally measured IMC growth curve (Fig. 1). During each time step, a thin shell of $\mathrm{Sn}$ ( $\sim 25 \mathrm{~nm}$ thick) surrounding the existing IMC nodules is transformed to IMC, simultaneously increasing the volume of IMC while consuming some of the Sn.

The spatial extent of the IMC is specified in the simulation by a field variable, $\Phi$, which is assigned a value between 0 and 1 at each node of the finite element mesh. $\Phi=0$ corresponds to $\mathrm{Sn}$, while $\Phi=1$ corresponds to IMC. Initially, all nodes within the Sn layer are assigned $\Phi=0$. During subsequent time steps, the value of $\Phi$ is updated at nodes which are to be transformed to IMC. The value of $\Phi$ at each node is determined by its distance, $r$, from the center of the nearest IMC nodule via a smoothed step function: $\Phi=1 / 2\left[1-\tanh \left(2\left(r-r_{\mathrm{IMC}}\right) / w\right)\right]$, where $w$ is the characteristic width of the IMC-Sn interface. Note that $\Phi$ varies from 0.98 to 0.02 between $r=\left(r_{\mathrm{IMC}}-w\right)$ and $r=\left(r_{\mathrm{IMC}}+w\right)$. Smoothing of the IMC-Sn interface prevents abrupt property changes as the interface passes through the finite element mesh. In the simulations, $w=0.1 \mu \mathrm{m}$.

The transformation of Sn to IMC is implemented in the finite element simulations by correlating material properties (stiffness, yield strength, molar volume) with the local value of $\Phi$. The material has the properties of Sn where $\Phi=0$ and the properties of IMC where $\Phi=1$. Property values are interpolated for intermediate values of $\Phi$. The molar volume change associated with the transformation is modeled by applying a stress-free transformation strain $^{25}$ corresponding to a $45 \%$ volumetric expansion. After application of the transformation strain, the material is in a state of compressive stress and would have to undergo a $45 \%$ expansion to become 


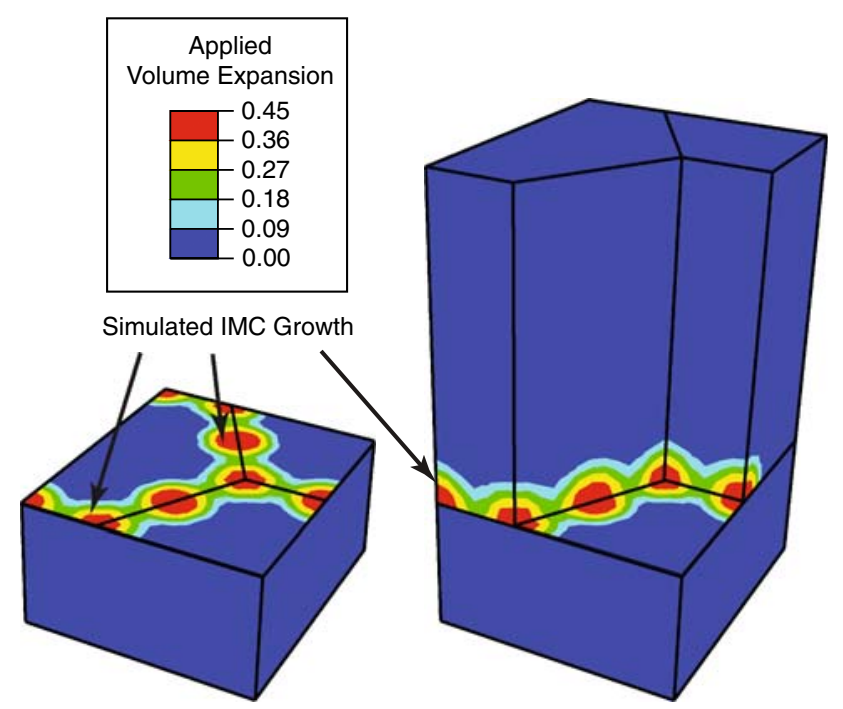

Fig. 3. IMC growth is simulated by applying a volumetric expansion to hemispherical regions distributed along the $\mathrm{Sn}-\mathrm{Sn}$ grain boundaries at the base of the $\mathrm{Sn}$ layer. The radius of the expanded regions is increased incrementally to model the progressive growth of the IMC. In the images shown, the corresponding IMC volume/area is $0.1 \mu \mathrm{m}$.

stress free. The transformation process is assumed to occur quasistatically, and stress equilibrium is maintained throughout the simulation.

To account for the elimination of grain boundaries within the transformed region, grain boundary diffusivity is scaled logarithmically with $\Phi$ via the relation $D_{\mathrm{gb}}^{*}=\epsilon^{\Phi} D_{\mathrm{gb}}$, where $D_{\mathrm{gb}}^{*}$ is the scaled diffusivity and $\epsilon$ is a finite constant chosen to be as small as possible without causing numerical problems in the solution. In the present case, $\epsilon=2 \times 10^{-10}$.

Finite element simulations were carried out using the ABAQUS software package, with specialized grain boundary diffusion elements implemented via a user-defined subroutine. For each simulation, mean biaxial stress through the entire Sn layer is computed as a volume weighted average of $\left(\sigma_{11}+\sigma_{22}\right) / 2$ taken over all unexpanded elements. This corresponds to the stress measured experimentally in the $\mathrm{Sn}$ via the wafer curvature technique described by Chason et al. ${ }^{13}$ In addition, we compute stress profiles through the Sn film along two vertical transects: one directly above a growing IMC nodule at a Sn-Sn-Sn triple junction, the other located at the center of a Sn grain. At each of 22 vertical positions through the film, stresses are averaged over all elements within a $0.15 \mu \mathrm{m}$ radius of the respective transect lines.

\section{RESULTS AND DISCUSSION}

The development of stress due to IMC growth has previously been explained in terms of elastic stress fields generated by IMC particles ${ }^{7,9}$ or combined elastic and plastic behavior within layers ${ }^{11}$ or around IMC particles. ${ }^{14}$ In addition, models for stress relaxation via whisker growth have included mass transport via grain boundary diffusion. ${ }^{6,11,17}$ However, the role of grain boundary diffusion in the development of stress within the Sn layer has not been quantitatively investigated. The primary goal of this paper is to determine which of the various assumptions regarding constitutive behavior of the Sn film best explains the experimental stress measurements. In the following discussion, we examine the consequences of three different combinations of constitutive behaviors: purely elastic deformation within Sn grains; elastic and plastic deformation within Sn grains; and elastic and plastic deformation within Sn grains combined with diffusion along grain boundaries. Contour plots of the mean biaxial stress, as well as stress profiles along vertical transects, resulting from different choices of constitutive behavior are presented in Fig. 4. The corresponding history of average Sn stress over time is plotted for each case in Fig. 5. We examine the meaning of the results for each case below.

We first consider the case of purely elastic behavior. The development of stress over time, shown in Fig. 5a, appears to mirror the growth of IMCs (curve in Fig. 1). The average stresses calculated in this case are an order of magnitude more compressive than those measured experimentally. Closer examination of the stress field in Fig. 4a and d reveals stresses of over $1000 \mathrm{MPa}$ immediately surrounding the IMC nodules. However, the stress field diminishes rapidly with distance from the IMC, and regions of both compressive and tensile in-plane stress are observed in the overlying Sn. Within the upper third of the Sn layer, the magnitude of the elastic stresses is only on the order of a few $\mathrm{MPa}$. Thus, if the Sn were capable of supporting stresses in excess of $1 \mathrm{GPa}$, a purely elastic mechanism for transmitting stress would result in little compressive stress distributed through the thickness of the film.

We next consider the case of elastic-plastic behavior within the $\mathrm{Sn}$ layer, but with no grain boundary diffusion. As discussed in the "Introduction," microscopy reveals significant dislocation activity within the Sn grains, providing direct evidence of plastic deformation. In the finite element analysis, a plastically deformed zone $\left(\sigma_{\text {mises }} \geq\right.$ 14.5 MPa in Fig. 6a) develops at the base of the Sn layer, around the growing IMC particles. Yield within the Sn allows the expansion of the IMC to be accommodated without generating the large stresses seen in the elastic case. Beyond the plastically deformed region, however, the stress field is elastic and therefore diminishes rapidly with distance from the IMC, as seen in Fig. 4b. In addition, regions of both compressive and tensile biaxial stress are observed in the Sn overlying the plastic zone.

As shown in Fig. 5b, the biaxial stress becomes steadily more compressive with continued IMC growth, reaching a value of $-6 \mathrm{MPa}$ after $150 \mathrm{~h}$. The gradual increase in compressive stress averaged through the Sn reflects the increasing fraction 

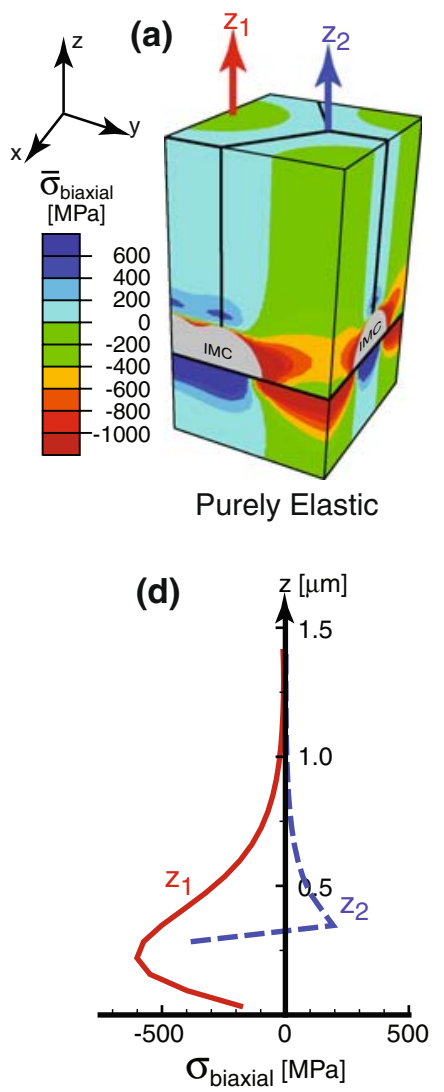
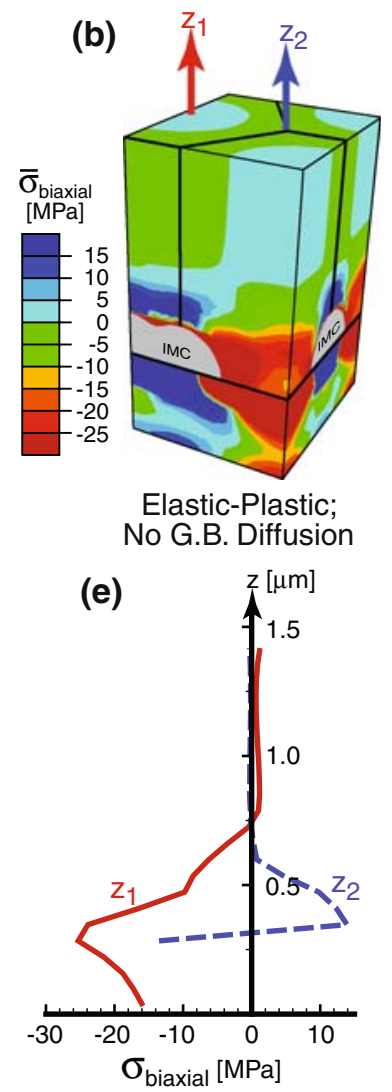
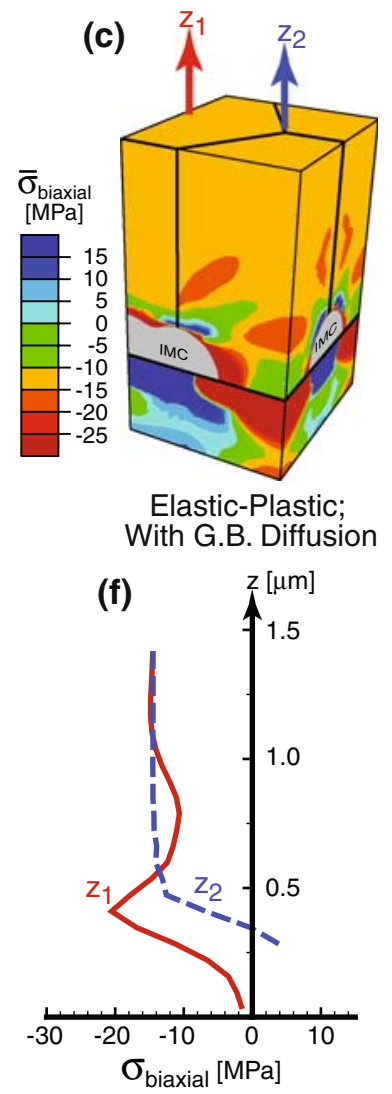

Fig. 4. Contours of mean biaxial stress through the film $(a-c)$ with stress profiles plotted for the same cases (d-e). Arrows labeled " $z_{1}$ " and " $z_{2}$ " on the contour plots indicate the locations of the vertical transects used to generate the stress profiles. Note that only the case including grain boundary diffusion $(\mathrm{c}, \mathrm{f})$ produces significant compressive stress through the $\mathrm{Sn}$ layer. In all cases, $t=40 \mathrm{~h}$ corresponding to total $V_{\text {IMC }} / A=0.13 \mu \mathrm{m}$

of the Sn layer that is at its yield stress. In contrast, the experimentally observed stress in the Sn reaches a maximum compressive value of $10 \mathrm{MPa}$ to $12 \mathrm{MPa}$ within the first $20 \mathrm{~h}$, then remains relatively constant.

Thus, while there is experimental evidence of dislocation-mediated plastic deformation within Sn grains, ${ }^{14}$ modeling indicates that elastic-plastic behavior alone is insufficient to explain the observed stress evolution associated with IMC growth. As in the case of purely elastic behavior, significant stresses develop in the immediate neighborhood of the IMC nodules, but die out very rapidly upward through the thickness of the film. As a result, very little compressive stress is transmitted through the thickness of the film-near the surface of the film, both tensile and compressive biaxial stresses are generated with magnitudes of less than $1 \mathrm{MPa}$.

Finally, we examine the stress that develops when IMC growth can be accommodated by mass transport through the Sn film via stress-driven grain boundary diffusion, in addition to elastic and plastic behavior within the Sn grains. In this case, the average biaxial stress in the Sn quickly reaches a maximum compressive value, then remains relatively constant as IMC growth continues (Fig. 5b). This results from the redistribution of volume along grain boundaries. Where compressive stresses normal to the grain boundaries are largest, in and around the plastic zone, $\mathrm{Sn}$ is removed from the adjacent grains and transported to less compressive regions of the grain boundary. Since the stress gradients are largely normal to the plane of the film, this results in net transport of volume from the base of the film toward the surface. As shown in Fig. 4c and $f$, the redistribution of volume via grain boundary diffusion produces relatively uniform compressive stress through the thickness of the Sn layer.

The transport of Sn along grain boundaries also affects the extent of the plastic deformation zone within the Sn grains. Contours of von Mises equivalent stress (Fig. 6a) show that, in the absence of grain boundary diffusion, the plastic zone $\left(\sigma_{\text {mises }} \geq\right.$ $14.5 \mathrm{MPa}$ ) is restricted to a narrow band at the base of the film, immediately surrounding the IMC particles. However, when grain boundary diffusion is active (Fig. 6b), the majority of the film is at or near the nominal yield stress. 

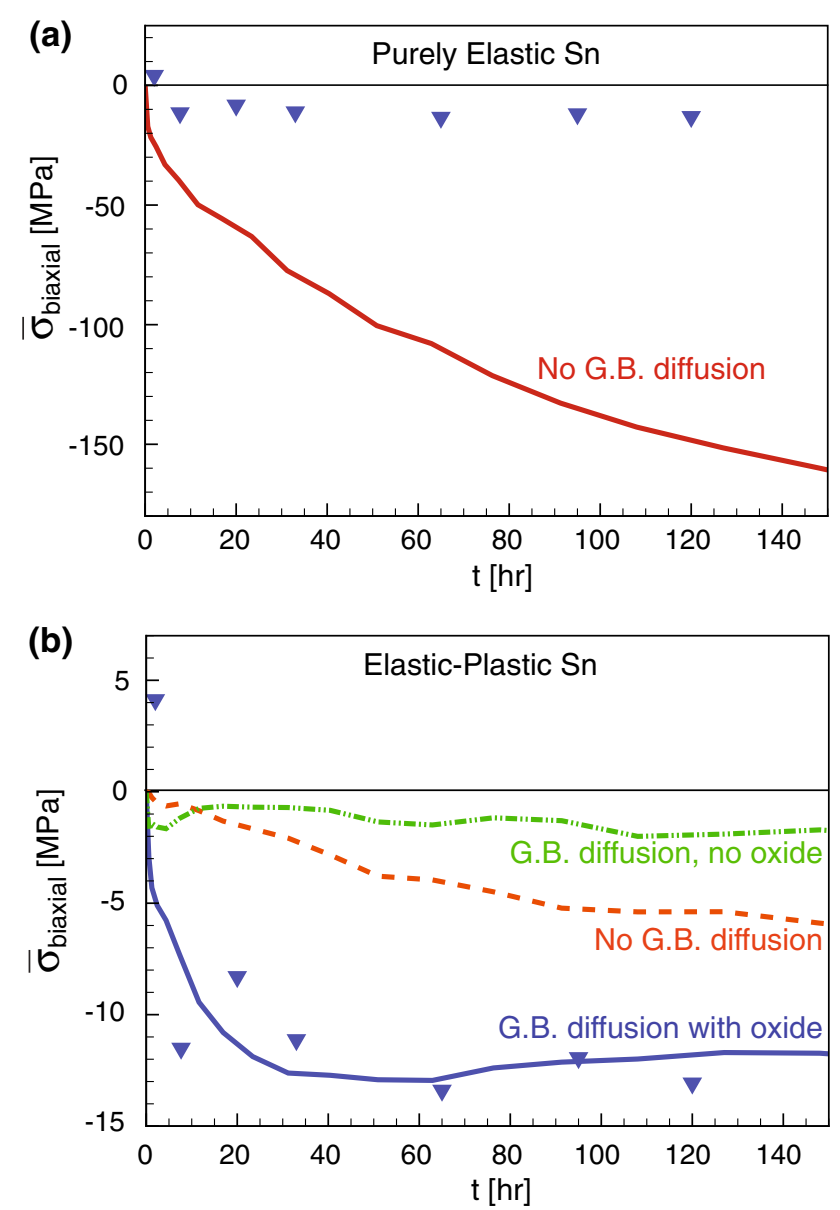

Fig. 5. Mean biaxial stress, averaged over the untransformed Sn. Symbols $(\boldsymbol{\nabla})$ are experimentally measured values; lines are simulation results. Results for the purely elastic case (a) are shown separately from elastic-plastic cases (b), as the stress levels differ by an order of magnitude. (a)

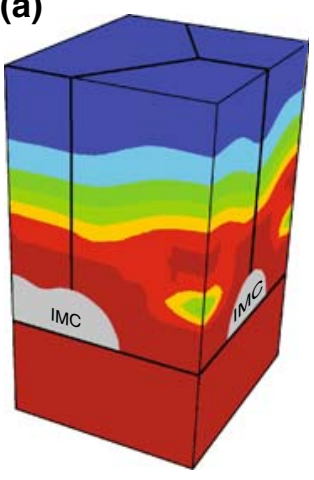

Elastic-Plastic; No G.B. Diffusion

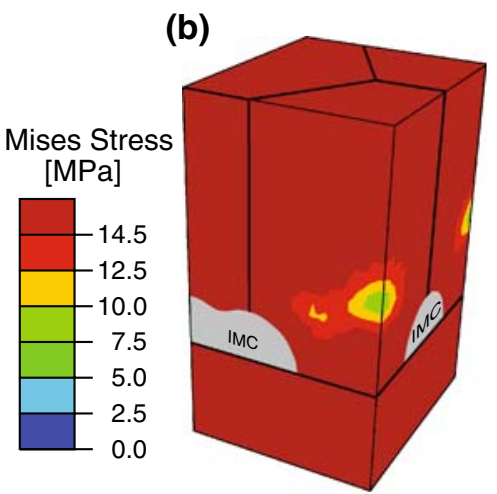

Elastic-Plastic: With G.B. Diffusion
Fig. 6. Contours of von Mises equivalent stress for simulations (a) without and (b) with diffusion along grain boundaries. In both cases, elastic-plastic constitutive behavior is assumed within the $\mathrm{Sn}$ grains. Without grain boundary diffusion (a), plastic yield ( $\left.\sigma_{\text {mises }} \geq 14.5 \mathrm{MPa}\right)$ is restricted to a band near the base of the film. However, when material can be transported via grain boundary diffusion (b) the plastically deforming region extends farther into the $\mathrm{Sn}$ and the entire film is near yield. $t=40 \mathrm{~h}$ and total $V_{\mathrm{IMC}} / A=0.13 \mu \mathrm{m}$ in both cases shown.
This mechanism for stress transmission helps explain why the measured stress in the Sn reaches a steady-state value which is relatively insensitive to further IMC growth. Only a small volume of IMC growth is required to drive sufficient transport of $\mathrm{Sn}$ upward through the film along grain boundaries and produce a relatively uniform stress state through the thickness of the film. After the stress in the Sn reaches the yield strength, additional IMC growth has little effect.

In our simulations, we assume that the transformation of Sn to IMC is accompanied by local volume expansion. As discussed in the "Experimental Background" section, another possibility is that Sn could be expelled to the grain boundaries during the reaction with little or no volume change in the transformed region. To evaluate this case, we consider the amount of volume that would have to be added to the grain boundaries to produce the experimentally observed stress. A biaxial strain of $2 \times 10^{-4}$ is sufficient to bring the Sn to its nominal yield stress of $14.5 \mathrm{MPa} \quad\left(\varepsilon=\left(1-v_{\mathrm{Sn}}\right) Y_{\mathrm{Sn}} / E_{\mathrm{Sn}} \approx\right.$ $\left.2 \times 10^{-4}\right)$. For a columnar film with a grain size $\phi=1.4 \mu \mathrm{m}$, this corresponds to a thickening of the grain boundaries by an amount, $\Delta \delta=\varepsilon \phi \approx 0.28 \mathrm{~nm}$, roughly equivalent to the addition of a monolayer of $\mathrm{Sn}$ atoms. Given the exceedingly small volume of excess Sn that would be required in the grain boundaries to produce uniform compressive stress in the $\mathrm{Sn}$, we conclude that IMC transformation involving Sn expulsion rather than (or in addition to) direct expansion is also a plausible mechanism for stress generation. Regardless of the details of the IMC transformation reaction, our primary conclusion, that grain boundary diffusion plays a central role in the development of stress within the film, remains unchanged.

The grain boundary diffusion mechanism for stress transmission that we have just demonstrated depends critically on two features of the Sn film: (1) columnar microstructure, and (2) the presence of a passivating oxide layer. Both are necessary to prevent relaxation of the stress-and therefore, their disruption may provide strategies for minimizing stress development within the Sn film.

The columnar microstructure of Sn films, with very few grain boundaries oriented parallel or oblique to the plane of the film, prevents stress relaxation via Coble creep. To summarize the argument given by Boettinger et al., ${ }^{11}$ grain boundaries parallel to the traction-free surface of the Sn would be subject to very small normal stress and thus have a lower diffusion potential relative to vertically oriented grain boundaries. This would drive mass transport from vertical to horizontal grain boundaries, resulting in reduced in-plane biaxial compression and slight overall thickening of the film. This raises an interesting question that could be answered by additional modeling: What fraction of parallel or oblique grain boundaries is sufficient to allow stress relaxation by Coble creep? 

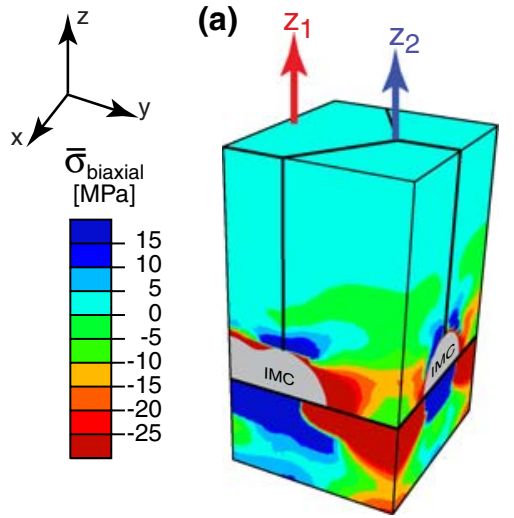

(b)

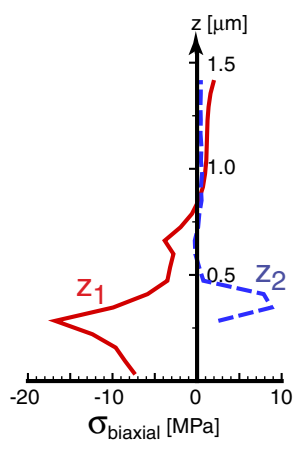

Fig. 7. When material is allowed to diffuse out of grain boundaries and across the free surface of the Sn, as would be the case if there were no passivating oxide layer present, IMC growth produces little stress through the thickness of the Sn. (a) Contours of mean biaxial stress at $t=40 \mathrm{~h}$, corresponding to total $V_{\mathrm{IMC}} / A=0.13 \mu \mathrm{m}$. (b) Stress profiles along vertical transects (marked by arrows $z_{1}$ and $z_{2}$ ) through the Sn layer.

The passivating oxide plays a similar constraining role. Without the oxide, diffusion of Sn out of the grain boundaries and onto the free surface of the $\mathrm{Sn}$ would be possible. Mass could be removed from grain boundaries with high compressive normal stress and transported to the traction-free surface of the Sn where the diffusion potential is much lower. Experimentally, in situ film stress measurements change by an amount consistent with the relaxation of compressive stress within the Sn layer when the surface oxide is removed by etching. ${ }^{13}$ To demonstrate the effect of the oxide in our finite element simulation, we eliminated the zero flux condition at the top of the grain boundaries which allows diffusion of material out onto the surface of the Sn. As seen in Figs. 5b and 7, removal of the surface oxide results in nearly complete relaxation of stress in the $\mathrm{Sn}$, except in the immediate vicinity of the IMCs.

We also tested the sensitivity of the modeled stress evolution to the value assumed for grain boundary diffusivity. Variation of the grain boundary diffusivity by two orders of magnitude above and below the literature value $\left(4.8 \times 10^{-11} \mathrm{~cm}^{2} / \mathrm{s} \leq D_{\mathrm{gb}} \leq 4.8 \times\right.$ $10^{-7} \mathrm{~cm}^{2} / \mathrm{s}$ ) produces a change of only about $10 \%$ in the computed average stress levels. From these results we conclude that the time scale governing grain boundary diffusion is much shorter than that governing IMC growth. Thus the development of stress in the Sn film is not limited by the rate of transport along grain boundaries, with the possible exception of the earliest phase of IMC formation when growth rates appear to be very fast.

The rapid time scale for grain boundary diffusion, together with the small excess volume within grain boundaries required to produce compressive stress, also implies that the average stress in the Sn should be relatively insensitive to the thickness of the film. As seen in Fig. 5c and f, relatively uniform biaxial compressive stress, near the yield strength, is produced in the Sn lying above the region of IMC growth. In simulations in which the film thickness is varied from $1.5 \mu \mathrm{m}$ to $9 \mu \mathrm{m}$ (with all other parameters identical) the average stress in the films varies by less than $10 \%$.

\section{CONCLUSIONS}

The growth of IMC particles at the base of a Sn film generates strain that must be accommodated by the surrounding Sn. Finite element simulations reveal that the details of the resulting stress field in the $\mathrm{Sn}$, as well as the average stress through the $\mathrm{Sn}$ layer, are strongly dependent on the deformation processes active within the Sn. Following is a summary of our conclusions.

1. Stress-driven grain boundary diffusion, coupled with elastic and plastic behavior within Sn grains, provides an effective mechanism for transmitting stress through the thickness of the Sn. Vertical stress gradients arising from the expansion of IMCs at the base of the Sn film drive mass transport upward along grain boundaries. The excess volume in the grain boundaries produces compressive stress resulting in relatively uniform stress though the entire thickness of the Sn.

2. This mechanism can explain why the stress in the Sn appears to quickly reach a "steady-state" value that remains constant even as IMCs continue to grow. Once sufficient volume has been driven into the grain boundaries to bring the entire film to yield, further IMC growth is accommodated plastically and produces no additional stress. The small excess grain boundary volume required to bring the film to yield and the fast rate of grain boundary diffusion relative to the rate of IMC growth helps explain how the measured Sn stress can reach its steady-state compressive value during the early stage of IMC growth.

3. The stress produced by elastic and plastic deformation within Sn grains, without fast transport of mass along grain boundaries, is largely concentrated in a region immediately surrounding the growing IMC particles, at the base of the Sn layer. Large stress gradients develop through the thickness of the film, as the stress field diminishes rapidly with distance from the IMC. In-plane stresses near the surface of the film have both tensile and compressive character and are only a few $\mathrm{MPa}$ in magnitude.

4. The effectiveness of grain boundary diffusion as a mechanism for transmitting compressive stress through the film depends on the columnar microstructure of the Sn, as well as the presence of a passivating oxide layer. If either of these could be disrupted, strain generated by the growth of IMCs could be relaxed via a Coble creep process without producing compressive stress in the $\mathrm{Sn}$, thus reducing the driving force for whisker growth. 


\section{ACKNOWLEDGEMENTS}

The authors gratefully acknowledge the support of the NSF through the Brown MRSEC (DMR 0079964) and helpful contributions from G. Barr.

\section{REFERENCES}

1. K.G. Compton, A. Mendizza, and S.M. Arnold, Corrosion 7, 327 (1951).

2. S.M. Arnold, Proceedings of 1959 Electronic Components Conference, Philadelphia, PA, May 6-8 (1959), pp. 75-82.

3. G.T. Galyon, Proceedings of SMTA International Conference, Chicago, IL, Sept. 26-30 (2004).

4. R.M. Fisher, L.S. Darken, and K.G. Carroll, Acta Metall. 2, 368 (1954).

5. K.N. Tu, Phys. Rev. B 49, 2030 (1994)

6. K.N. Tu, Mater. Chem. Phys. 46, 217 (1996).

7. B.Z. Lee and D.N. Lee, Acta Metall. 46, 3701 (1998).

8. W.J. Choi, T.Y. Lee, K.N. Tu, N. Tamura, R.S. Celestre, A.A. MacDowell, Y.Y. Bong, and L. Nguyen, Acta Metall. 51, 6253 (2003).

9. R. Krishnamurthy and D.J. Srolovitz, Acta Metall. 53, 5189 (2005)

10. K.N. Tu and J.C.M. Li, Mater. Sci. Eng. A 409, 131 (2005).

11. W.J. Boettinger, C.E. Johnson, L.A. Bendersky, K.-W. Moon, M.E. Williams, and G.R. Stafford, Acta Metall. 53, 5033 (2005).

12. G.T. Galyon and L. Palmer, IEEE Trans. Electron. Packag. Manuf. 28, 17 (2005).
13. E. Chason, N. Jadhav, W.L. Chan, L. Reinbold, and K.S. Kumar, Appl. Phys. Lett. 92, 171901 (2008).

14. K.S. Kumar, L. Reinbold, A.F. Bower, and E. Chason, J. Mater. Res. 23, 2916 (2008)

15. W.F. Gale and T.C. Totemeier, eds., Smithells Metals Reference Book, 8th ed. (Burlington, MA: Elsevier Butterworth-Heinemann, 2004).

16. G.T.T. Sheng, C.F. Hu, W.J. Choi, K.N. Tu, Y.Y. Bong, and L. Nguyen, J. Appl. Phys. 92, 64 (2002).

17. B. Hutchinson, J. Oliver, M. Nylén, and J. Hagstroem, Mater. Sci. Forum 467-470, 465 (2004).

18. W. Zhang, A. Egli, F. Schwager, and N. Brown, IEEE Trans. Electron. Packag. Manuf. 28, 85 (2005).

19. K.N. Tu and R.D. Thompson, Acta Metall. 30, 947 (1982).

20. L.B. Freund and S. Suresh, Thin Film Materials (Cambridge: Cambridge University Press, 2003).

21. R.J. Fields, S.R. Low III, and G.K. Lucey Jr., Metal Science of Joining, ed. M.J. Cieslak, J.H. Perepezko, S. Kang, and M.E. Glicksman (Warrendale, PA: The Minerals, Metals \& Materials Society, 1992), p. 165.

22. X. Deng, N. Chawla, K.K. Chawla, and M. Koopman, Acta Metall. 52, 4291 (2004).

23. W. Lange and D. Bergner, Phys. Stat. Sol. 2, 1410 (1962).

24. A.F. Bower and E. Wininger, J. Mech. Phys. Solids 52, 1289 (2004).

25. The technique employed here is the same as that typically used to model thermal expansion. 